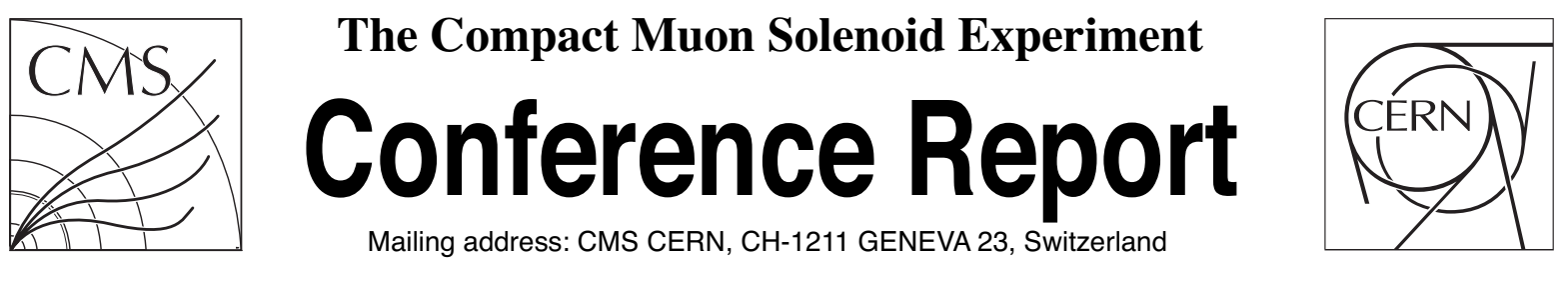

03 July 2015 (v3, 21 July 2015)

\title{
Longevity of the CMS ECAL and scintillator-based options for electromagnetic calorimetry at HL-LHC
}

Hengne Li for the CMS Collaboration

\begin{abstract}
The CMS lead tungstate $\left(\mathrm{PbWO}_{4}\right)$ electromagnetic calorimeter (ECAL) has successfully achieved its first goal, the Higgs discovery 2012. However, longevity studies show that part of the $\mathrm{PbWO}_{4}$ ECAL does not maintain the required performance due to the radiation damaged incurred at the HL-LHC. The forward region of the detector will suffer the most from radiation damage, and the ECAL Endcaps (EE) will need to be replaced. A scintillator-based option for the EE replacement, Shashlik, is presented in the paper. The Shashlik EE is a sampling calorimeter. Tungsten absorber plates are interleaved with scintillator plates (LYSO or $\mathrm{CeF}_{3}$ ), with quartz and wavelength-shifting (WLS) capillaries optically coupled to the scintillator plates for light output. The Shashlik EE maintains a high energy resolution, but compared to the current $\mathrm{PbWO}_{4} \mathrm{EE}$, it is at least five times greater in radiation hardness and has a module size four times smaller allowing four times higher granularity laterally for pileup mitigation and particle identification. Irradiation tests and beam tests results have confirmed the high performance of the Shashlik EE.
\end{abstract}

Presented at SCINT2015 13th International Conference on Inorganic Scintillators and their Applications 


\title{
Longevity of the CMS ECAL and Scintillator-Based Options for Electromagnetic Calorimetry at HL-LHC
}

\author{
Hengne Li
}

\begin{abstract}
The CMS lead tungstate $\left(\mathrm{PbWO}_{4}\right)$ electromagnetic calorimeter (ECAL) has successfully achieved its first goal: the discovery of the Higgs boson in 2012. However, longevity studies show that part of the $\mathrm{PbWO}_{4}$ ECAL will not maintain the required performance due to radiation damage incurred at the HL-LHC. The forward region of the detector will suffer the most from radiation damage, and the ECAL Endcaps (EE) will need to be replaced. A scintillator-based option for the EE replacement, Shashlik EE, is presented in the paper. The Shashlik EE is a sampling calorimeter. Tungsten absorber plates are interleaved with scintillator plates (LYSO or $\mathrm{CeF}_{3}$ ), with quartz and wavelength-shifting (WLS) capillaries optically coupled to the scintillator plates for light output. The Shashlik EE maintains an excellent energy resolution, but compared to the current $\mathrm{PbWO}_{4}$ EE, it is at least five times greater in radiation hardness and has a module size four times smaller allowing four times higher granularity laterally for pileup mitigation and particle identification. Irradiation tests and beam tests results have confirmed the high performance of the Shashlik EE.
\end{abstract}

Index Terms-Calorimetry, photondetectors, solid scintillation detectors.

\section{Introduction: CurRent CMS ECAL IS A Big SUCCESS}

T HE CMS [1] electromagnetic calorimeter (ECAL) is a homogeneous crystal calorimeter, made of 75,848 lead tungstate $\left(\mathrm{PbWO}_{4}\right)$ scintillating crystals. The ECAL consists of a Barrel region, covering the pseudorapidity range $|\eta|<1.48$, which is closed by two Endcaps that extend the coverage up to $|\eta|=3$. The choice of $\mathrm{PbWO}_{4}$ crystals provides high radiation tolerance, a small radiation length $(0.89 \mathrm{~cm})$ and Molière radius $(2.19 \mathrm{~cm})$, and fast scintillation response. These properties have made it possible to build a compact and granular homogeneous calorimeter with excellent energy resolution, response linearity and particle identification capability at the LHC [2].

During LHC Run I, the CMS detector has recorded data with instantaneous luminosities close to the design goal of $1 \times 10^{34} \mathrm{~cm}^{-2} \mathrm{~s}^{-1}$, with an average number of simultaneous interactions per bunch crossing (pileup) of about 25 . The excellent performance of the CMS ECAL in this environment has led to the discovery of a Higgs boson in 2012 [3].

Manuscript received July 20, 2015; revised October 15, 2015; accepted November 16, 2015. Date of current version April 15, 2016.

The author is with the (On behalf of the CMS collaboration) Department of Physics, University of Virginia, Charlottesville, VA 22904-4714 USA (e-mail: Hengne.Li@cern.ch).

Color versions of one or more of the figures in this paper are available online at http://ieeexplore.iee.org.

Digital Object Identifier 10.1109/TNS.2016.2524622

\section{Opportunities And Challenges AT HL-LHC}

The HL-LHC upgrade, planned for 2025, will significantly increase the instantaneous luminosity that can be provided to CMS, with a total integrated luminosity of $3000 \mathrm{fb}^{-1}$ expected to be delivered by 2035. It has been shown [4] that the significantly larger dataset provided by the HL-LHC can considerably expand the sensitivity and reach of CMS to various key physics signatures, including the precise measurement of Higgs boson couplings (including VBF processes), searches for supersymmetry and heavy vector gauge bosons, and precise measurements of electroweak processes. However, the HL-LHC performance criteria require a re-examination of the ability of the detector active material and readout to meet these requirements up to an integrated luminosity of $3000 \mathrm{fb}^{-1}$.

ECAL lead tungstate crystals were optimized for their application in CMS, particularly with respect to radiation hardness through many studies with ionizing electromagnetic radiation and hadrons [5]. The dominant concern for the HL-LHC is the loss of response due to hadron irradiation, which is cumulative and does not anneal at room temperature as is the case for gamma radiation. In CMS the flux of hadrons is dominated by charged pions with energies of order $1 \mathrm{GeV}$. The effect of charged hadrons has been studied with crystals irradiated with pions and protons from the CERN Proton Synchrotron.

Recent and extensive beam tests of hadron-irradiated crystals have been performed to determine the expected light transmission and energy resolution of the calorimeter during HL-LHC operation. Based on these results, a model of crystal ageing has been developed and used to simulate the expected performance of ECAL at HL-LHC luminosities. Fig. 1 shows the predicted response reduction, $\mathrm{S} / \mathrm{S}_{0}$, for an electron shower of $50 \mathrm{GeV}$ as a function of pseudorapidity for various assumed integrated luminosities [6]. Fig. 2 shows a special high pileup event taken in 2012 with 78 reconstructed vertices [6]. The expected average number of vertices at HL-LHC is about 140 . The consequences of this loss of response and the large number of pileup on electron and photon energy resolution and identification are summarized in [6].

The conclusion is that the ECAL Barrel will still perform well with some modifications, while the ECAL Endcaps need to be replaced for HL-LHC operation.

\section{ECAL BARREL UPGRADE}

The ECAL Barrel upgrade is driven by the requirements for a trigger latency of $12.5 \mu$ s and a Level-1 trigger rate of $750 \mathrm{kHz}$. We will use this opportunity to also optimize the electronics and 


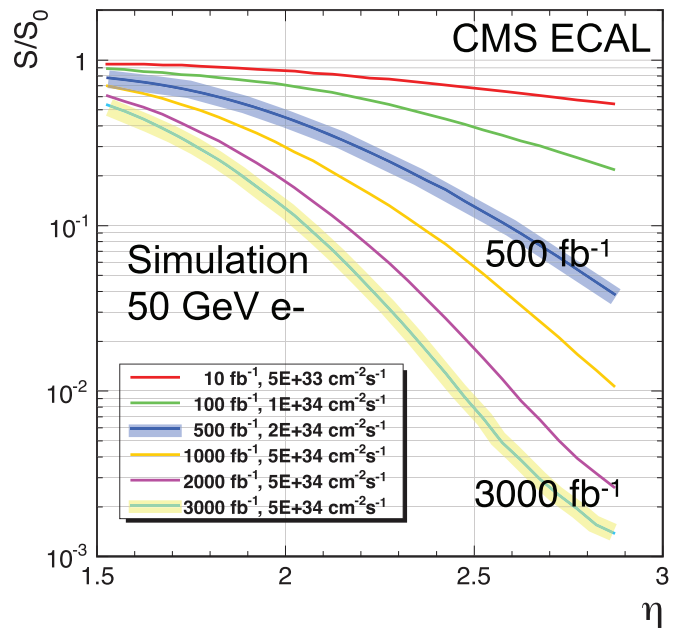

Fig. 1. Prediction of the relative response, $S / \mathrm{S}_{0}$, measured in the ECAL Endcaps for a $50 \mathrm{GeV}$ electron shower, plotted as a function of pseudorapidity $\eta$ for a range of integrated luminosities up to $3000 \mathrm{fb}^{-1}$.

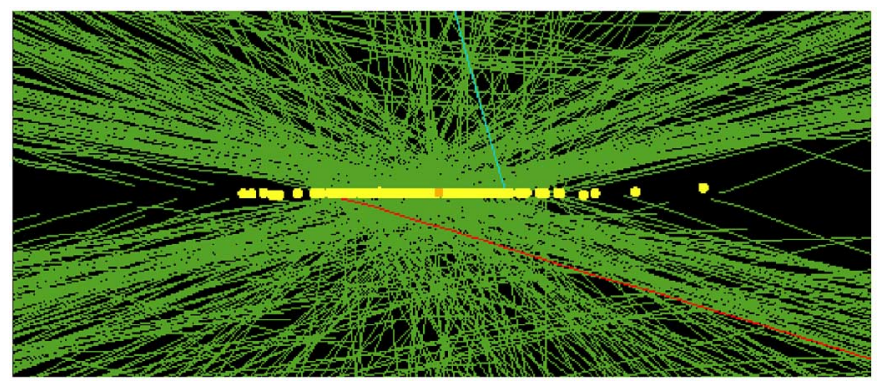

Fig. 2. High pileup event with 78 reconstructed vertices recorded in 2012.

the operating temperature of the system to mitigate the increase in noise of the avalanche-photodiodes (APDs) caused by the LHC irradiation. Fig. 3 shows the expected noise as a function of integrated luminosity for different operating temperatures and pulse shaping times [6]. One can see a $35 \%$ reduction of the noise by reducing the operating temperature from 18 to $8{ }^{\circ} \mathrm{C}$, and another $30 \%$ reduction in noise by shortening the pulse shaping time from $\tau=43$ ns to 20 ns with upgraded electronics. Fig. 4 shows the expected energy resolution before and after the ECAL Barrel upgrade [6]. The upgraded energy resolution with an average of 140 vertices from pileup after $1000 \mathrm{fb}^{-1}$ is expected to be as good as the resolution with 50 vertices from pileup without aging.

\section{ECAL ENDCAP UPGRADE OPTION: SHASHLIK}

\section{A. Shashlik Design}

One of the options for the upgrade of the ECAL Endcap was a Shashlik design. The Shashlik ECAL Endcap is a sampling calorimeter with tungsten as absorber and LYSO or $\mathrm{CeF}_{3}$ crystals as the scintillator. Fig. 5 shows a single Shashlik module with LYSO crystals. The module front face has a size of $1.4 \times 1.4 \mathrm{~cm}^{2}$, and a length of $11.4 \mathrm{~cm}$. The tungsten and scintillator plates are interleaved with each other, using four quartz wavelength shifting (WLS) capillaries to guide the scintillation

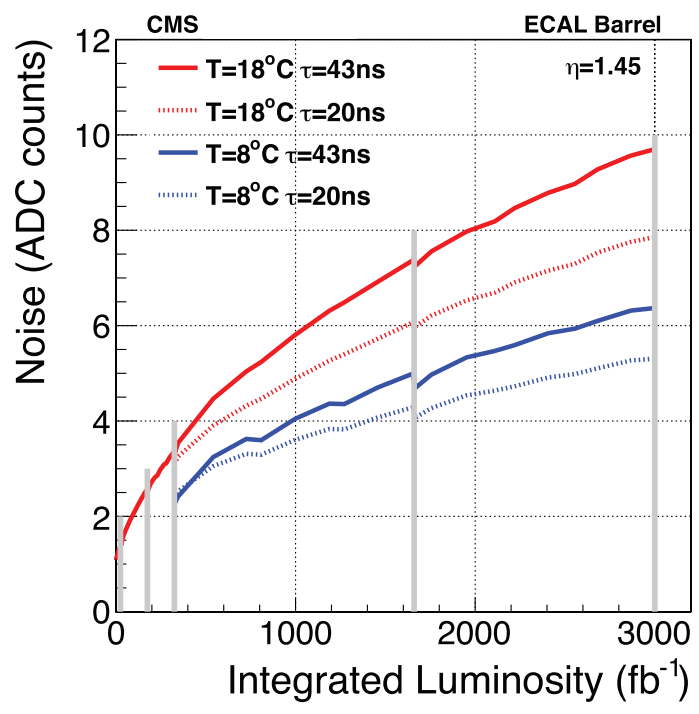

Fig. 3. Expected noise level in the ECAL Barrel as a function of integrated luminosity at $|\eta|=1.45$, for different operating temperatures and pulse shaping times.

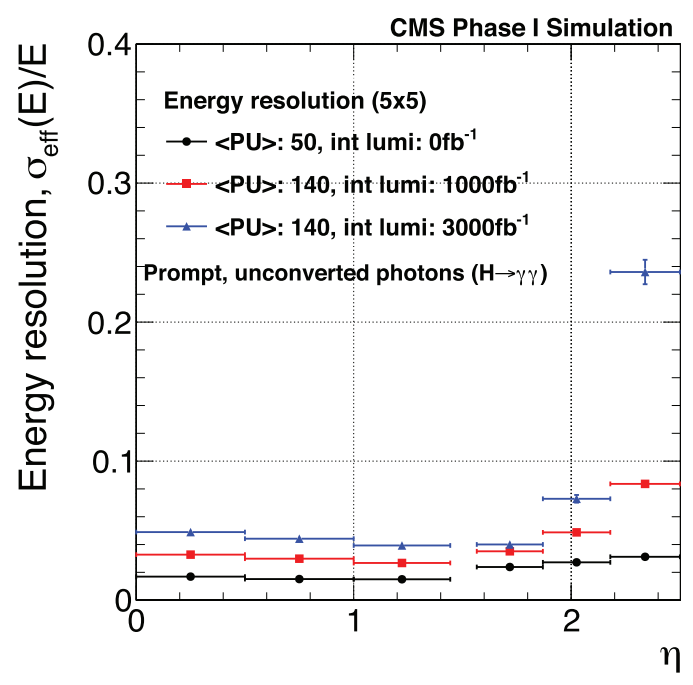

Fig. 4. Energy resolution for photons from Higgs boson decays into $\gamma \gamma$ for different integrated luminosity and pileup scenarios, comparing before and after the ECAL Barrel upgrade.

light outside the scintillator plates. The WLS dye is proprietary and is called "DSB1". Fig. 6 illustrates how scintillation light from scintillator plates is collected through the WLS capillaries. The capillaries guide the light outside the detector to silicon photomultipliers (SiPM) to reduce the radiation damage to the SiPMs. ${ }^{1}$ Fig. 7 illustrates the geometry of the Shashlik ECAL Endcap inside CMS, showing how the capillaries guide the light outside the detector. The charge integrator and encoder (QIE) developed at Fermilab [7] is used as the front-end readout card with one channel per module. Fig. 8 shows photos of a prototype module of the Shashlik (LYSO) that was used in the CERN beam test in October 2014.

\footnotetext{
${ }^{1}$ A new radiation-hard photodetector using Gallium Indium Phosphide (GaInP) instead of silicon is being developed.
} 


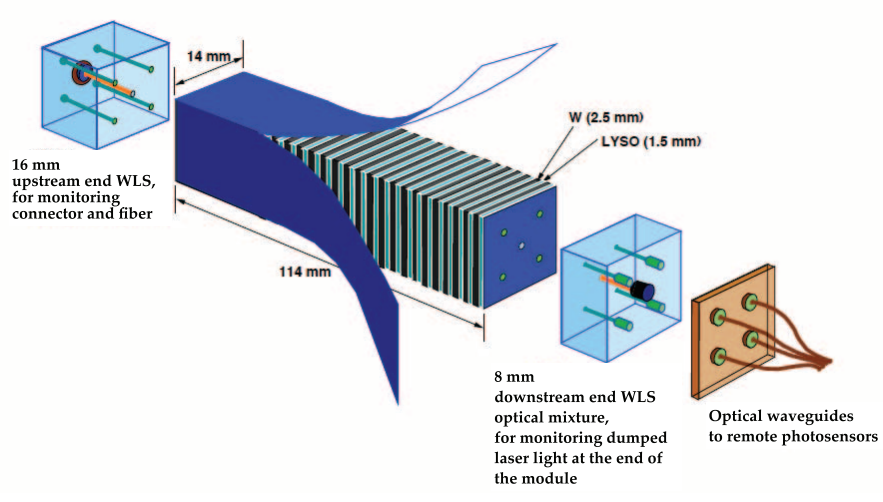

Fig. 5. Design of a single Shashlik module using LYSO as scintillator.

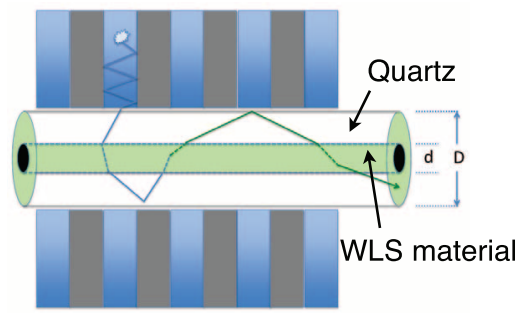

\section{Capillary concept}

Fig. 6. Idea of the quartz WLS capillary design. Scintillation light from a scintillator plate is collected by a quartz WLS capillary that plugged in a Shashlik module.

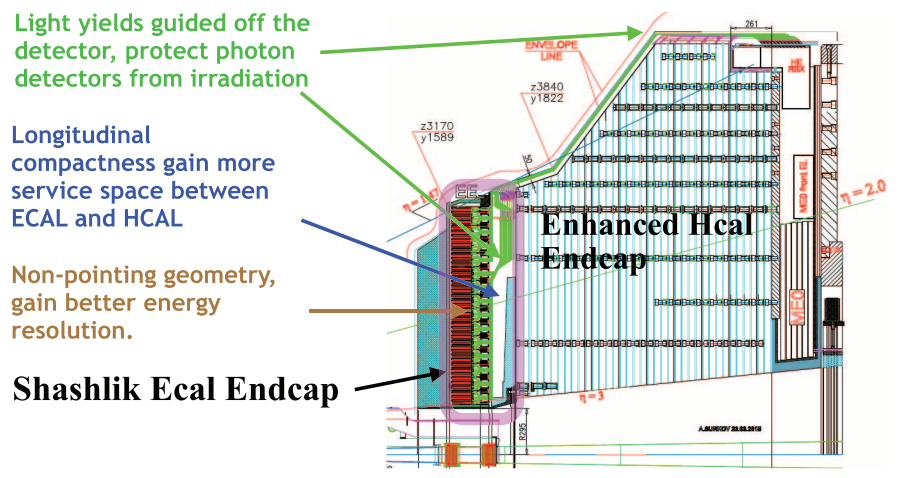

Fig. 7. Geometry scheme of the Shashlik ECAL Endcap. The dimensions are in $\mathrm{mm}$.

\section{B. Key Features}

In order to understand better the Shashlik design, Table I compares some key parameters between the Shashlik (LYSO) Endcap and the current $\mathrm{PbWO}_{4}$ ECAL.

First of all, the Shashlik is two times more dense longitudinally compared to the $\mathrm{PbWO}_{4}$ ECAL. One motivation for the compactness in depth is to have less radiation damage affecting the light collection using the WLS capillaries, because the length of the capillaries inside a module is shorter. Another reason for the compactness is to have more service space behind the ECAL Endcap (see Fig. 7).

Second, the Shashlik is about two times more dense laterally, as given by the average Molière radius, and thus allows four
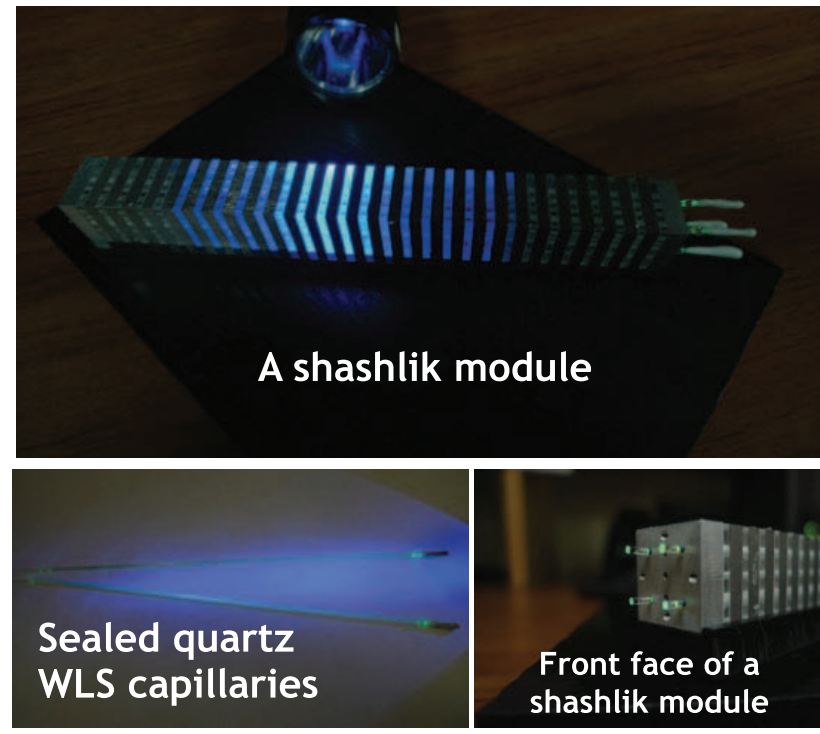

Fig. 8. Photos of Shashlik module and capillaries.

TABLE I

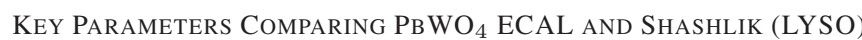

\begin{tabular}{c|c|c}
\hline & PbWO $_{4}$ & W/LYSO $(\mathrm{Ce})$ \\
\hline Average radiation length $\mathrm{X}_{0}(\mathrm{~mm})$ & 8.9 & 4.8 \\
\hline Length $(\mathrm{mm})$ & $220\left(25 \mathrm{X}_{0}\right)$ & $114\left(24 \mathrm{X}_{0}\right)$ \\
\hline Sampling fraction & homogeneous & $18 \%$ \\
\hline Average Molière radius $(\mathrm{mm})$ & 21.0 & 13.7 \\
\hline Transverse size $(\mathrm{mm})$ & 28.6 & 14.0 \\
\hline Number of modules for 2 Endcaps & 14648 & 60800 \\
\hline Light yield (relative to Nal) & $0.3 \%$ & $85 \%$ \\
\hline Module light output of $(\mathrm{p} . \mathrm{e} . / \mathrm{MeV})$ & 4.5 & 6.0 \\
\hline Temperature dependence $\left(\% /^{\circ} \mathrm{C}\right)$ & -2.2 & -0.2 \\
\hline
\end{tabular}

times higher lateral granularity. The high granularity is a key feature for pileup mitigation; for example, one can have better electromagnetic cluster and hadron cluster separation with higher granularity.

Third, LYSO has two orders of magnitude higher light yield than $\mathrm{PbWO}_{4}$. This is an essential feature of the Shashlik design, where we interleave the LYSO and tungsten plates, while only LYSO is transparent and can generate scintillation light. We observe that the Shashlik LYSO can produce an equal or higher light output per module compared to the homogeneous $\mathrm{PbWO}_{4}$ ECAL.

Lastly, the temperature dependence of the light yield is much smaller for LYSO than for $\mathrm{PbWO}_{4}$.

\section{Radiation Hardness Test Results}

Many studies have been pursued to measure the radiation hardness of the Shashlik design. Simulation studies and irradiation tests [8] show that LYSO and $\mathrm{CeF}_{3}$ are more radiation resistant than $\mathrm{PbWO}_{4}$. As shown in Fig. 9, LYSO has a factor of five lower proton damage with respect to $\mathrm{PbWO}_{4}$. In the figure, the effect of the proton irradiation is measured in terms of the coefficient of induced absorption, as defined below

$$
\mu_{\mathrm{IND}}(\lambda)=-\frac{1}{L} \ln \left(\frac{T(\lambda)}{T_{0}(\lambda)}\right)
$$




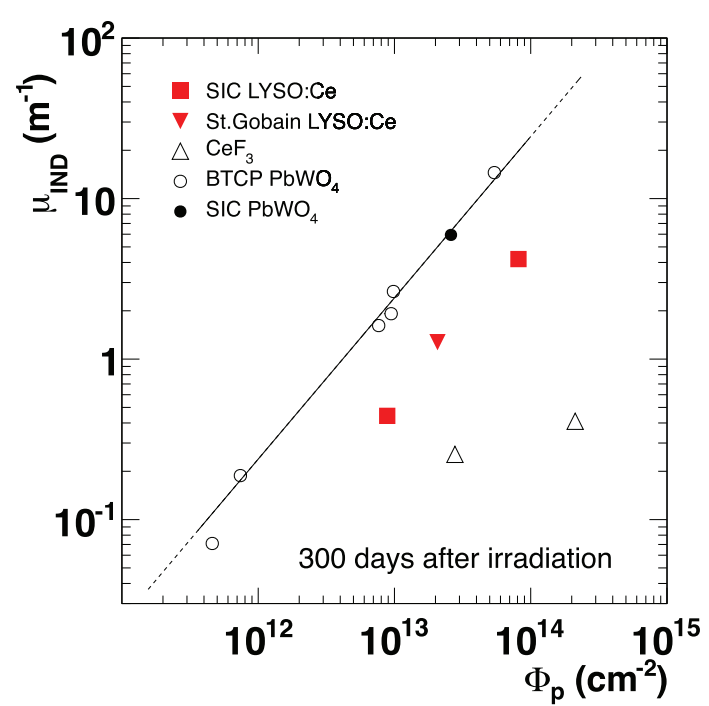

Fig. 9. Induced absorption coefficient $\left(\mu_{I N D}\right)$ as a function proton fluence $\left(\Phi_{p}\right)$ for $\mathrm{PbWO}_{4}, \mathrm{LYSO}(\mathrm{Ce})$, and $\mathrm{CeF}_{3}$ crystals [8].

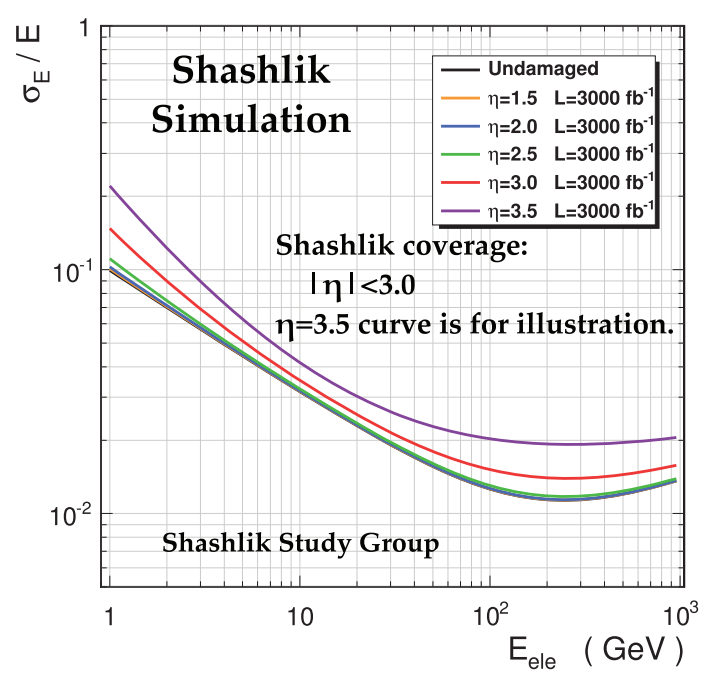

Fig. 10. Expected energy resolution of Shashlik ECAL as a function of electron energy at different $\eta$. The $|\eta|=3.5$ curve is for illustration only, since the Shashlik $|\eta|$ coverage is up to 3.0 [9].

where $T$ is the attenuation of the light transmission along the length $L$ at a specific wavelength $\lambda$ with respect to its initial value, $T_{0}$.

As shown in Fig. 10, Monte Carlo simulations [9] of single electrons (including a model of the hadron irradiation damage) show that the degradation on electromagnetic energy resolution is insignificant after $3000 \mathrm{fb}^{-1}$ of integrated luminosity for the designed Shashlik $\eta$ coverage $|\eta|<3.0$.

The WLS capillaries were also tested for radiation hardness [9]. The test was performed at Los Alamos National Laboratory using a beam of $800 \mathrm{MeV}$ protons. Three capillaries were used in the test, with two of them irradiated and one kept as a reference. The proton radiation dosage is $2.7 \times 10^{14}$ protons $/ \mathrm{cm}^{2}$ at $800 \mathrm{MeV}$.

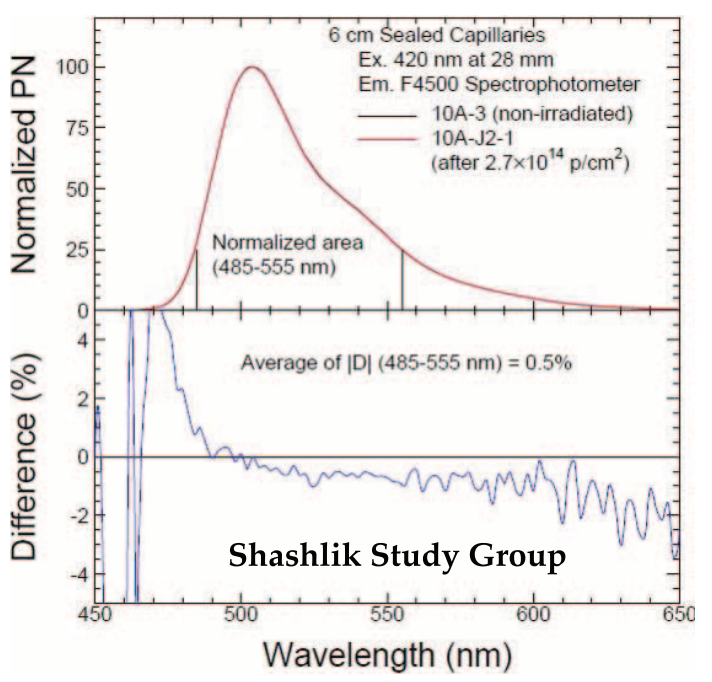

Fig. 11. Wavelength emission spectra of the quartz WLS capillaries comparing irradiated capillaries and non-irradiated capillaries [9].

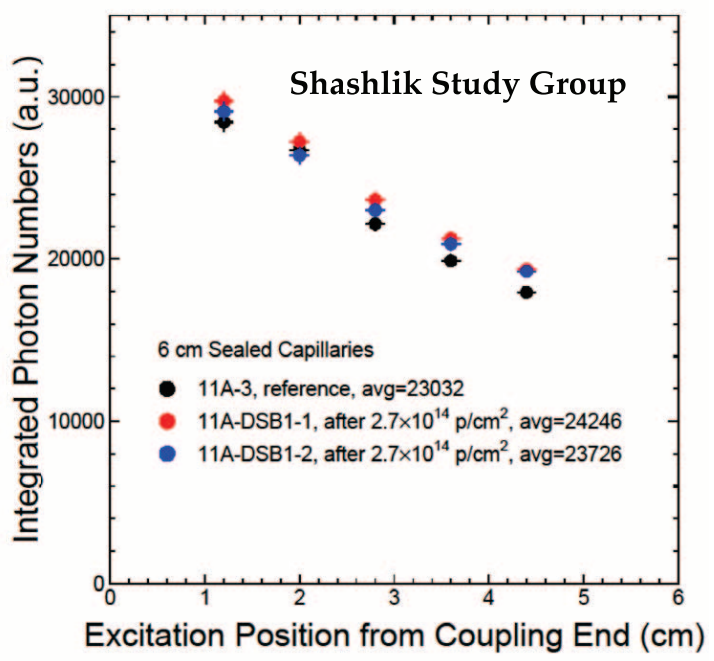

Fig. 12. Integrated number of photons collected as a function of excitation position from the coupling end of the quartz WLS capillaries comparing irradiated capillaries and non-irradiated capillaries [9].

The irradiated capillaries were then measured at Caltech. In Fig. 11 we see that there is no discernible difference in the photon emission spectra comparing irradiated and non-irradiated capillaries. In Fig. 12, the light yield as a function of excitation position in the capillaries also shows only negligible differences between irradiated and non-irradiated capillaries.

\section{Beam Test Results}

The design goal for the energy resolution of the Shashlik ECAL is a $10 \%$ stochastic term, and $1 \%$ constant term as shown in the following expression of the energy resolution:

$$
\frac{\sigma(E)}{E}=\frac{10 \%}{\sqrt{E(\mathrm{GeV})}} \oplus \frac{100(\mathrm{MeV})}{E(\mathrm{GeV})} \oplus 1 \% \text {. }
$$

The first term on the right hand side of the equation is the stochastic term, which represents uncertainties from sampling 


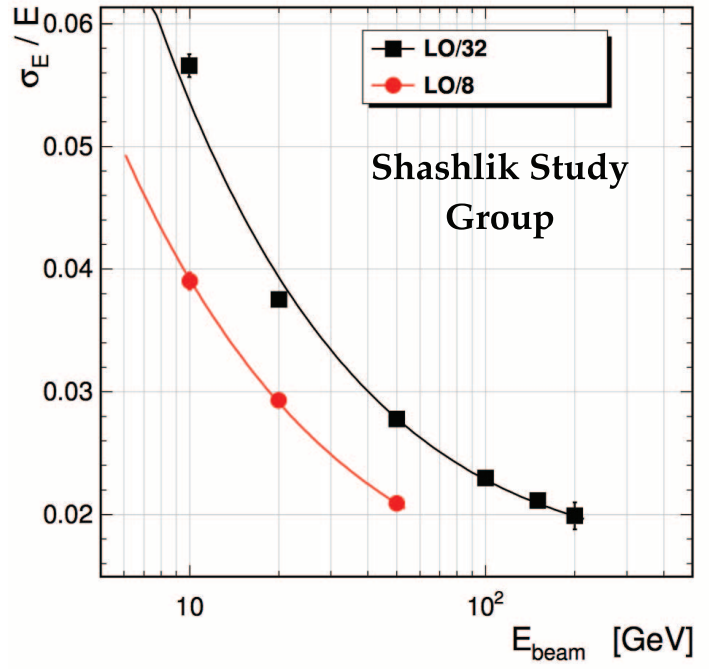

Fig. 13. Energy resolution results from Shashlik (LYSO) CERN beam test in October 2014 [9]. The energy resolution is measured with two levels of light yield attenuation, LO/32 (light output divided by 32 ) and LO/8.

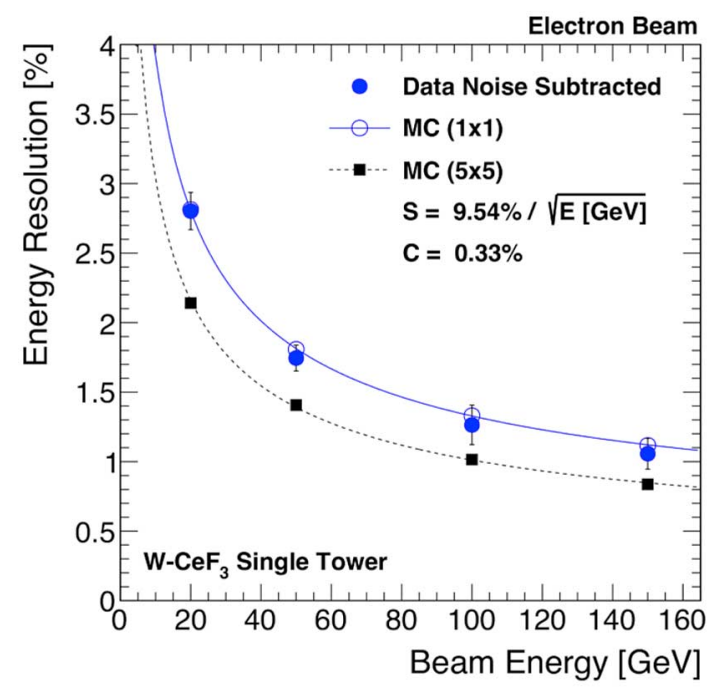

Fig. 14. Energy resolution results from Shashlik $\left(\mathrm{CeF}_{3}\right) \mathrm{CERN}$ beam test in October 2014 [10].

fraction, photon statistics, and transverse containment. The second term is the noise term, which quantifies electronics and pileup noise. And the third term, the constant term, describes the uncertainties from inter-calibration, longitudinal containment, and transverse uniformity.

The results [9] shown in Figs. 13 and 14 are from 2014 beam tests at CERN, for LYSO and $\mathrm{CeF}_{3}$, respectively. The LYSO prototype used in the beam test is composed of a $4 \times 4$ array of the Shashlik modules presented in Fig. 5. For the LYSO results, the two colors are for the two levels of light yield attenuation using a neutral density (ND) filter ${ }^{2}$. In Fig. 12, "LO/32" means "light output divided by a factor of 32", namely 32 times light yield attenuation, and "LO/8" means 8 times light yield

\footnotetext{
${ }^{2}$ The reason to do the light yield attenuation is simply because the LYSO intrinsic light yield is too high that the SiPMs used in the beam tests (having larger pixel size than that envisaged for the final device) are saturated in the absence of attenuation.
}

TABLE II

Stochastic TERM EXPECTEd (STANDAlone Simulation) AND MEAsured From THE SHASHLIK LYSO PROTOTYPE AT CERN IN OCTOBER 2014 [9]

\begin{tabular}{c|c|c}
\hline ND Filter & Expected & Measured \\
\hline None & $10.1 \%$ & \\
\hline LO/8 & $11.3 \%$ & $11.7 \%$ \\
\hline LO/32 & $15.6 \%$ & $15.8 \%$ \\
\hline
\end{tabular}

attenuation. For the $\mathrm{CeF}_{3}$ results in Fig. 13, the $\mathrm{MC}$ simulation assumes a stochastic term of $10 \%$ for a cluster fully contained by the calorimeter. The simulation results, which are for a single module $(1 \times 1)$ and a cluster of $5 \times 5$ modules, are compared with the beam test data result using a single module $(1 \times 1)$. We can see that the single module results from beam test data and MC simulation are in good agreement. Please see Ref [10] for details about the $\mathrm{CeF}_{3}$ results.

Table II shows the measured stochastic term with the two levels of light yield attenuation compared with the expected values from standalone simulation assuming a $10 \%$ stochastic term in case of no light yield attenuation. We can see good agreement between expectation and beam test measurements.

\section{Conclusion}

The current $\mathrm{CMS} \mathrm{PbWO}_{4}$ ECAL has achieved its excellent performance during LHC Run I [2]. However, for the HLLHC, the harsh radiation environment presents challenges that result in significant radiation damage in the forward regions of the detector. Studies show that the ECAL Barrel can perform well with some modifications, but the Endcaps will need to be replaced. For the Endcap, the Shashlik design is an excellent option which uses radiation-hard scintillators and WLS capillaries. The Shashlik ECAL Endcap is two times more compact longitudinally, which allows for less radiation damage impacting the light collection and also more service space behind ECAL for guiding the light output outside the detector to protect the photodetectors from irradiation. It is also two times more dense laterally, and thus four times higher granularity laterally for pileup mitigation and particle identification. Beam test results confirm the Shashlik design energy resolution with a stochastic term of $10 \%$.

The author would like to thank the Shashlik Study Group [9] for the excellent design of the Shashlik ECAL Endcap and institutes supporting experimental facilities for the beam tests and irradiation tests: CERN, Los Alamos, Caltech, and Fermilab.

\section{REFERENCES}

[1] CMS Collaboration, "The CMS experiment at the CERN LHC," JINST vol. 3, p. S08004, 2008

[2] CMS Collaboration, "Energy calibration and resolution of the CMS electromagnetic calorimeter in pp collisions at $\sqrt{s}=7 \mathrm{Tev}$," JINST, vol. 8, p. 09009, 2013.

[3] CMS Collaboration, "Observation of a new boson at a mass of $125 \mathrm{GeV}$ with the CMS experiment at the LHC," Phys. Lett. B, vol. 716, no. 1 pp. 30-61, 2012.

[4] CMS Collaboration, "Projected performance of an upgraded CMS detector at the LHC and HL-LHC: Contribution to the snowmass process," arXiv, vol. 1307, p. 7135. 
[5] P. Adzic et al., "Radiation hardness qualification of PbWO4 scintillation crystals for the CMS Electromagnetic Calorimeter," JINST, vol. 5, p. 03010, 2010, references therein.

[6] CMS Collaboration, "Technical proposal for the phase-II upgrade of the CMS detector," CERN-LHCC-2015-010; LHCC-P-008, 2015.

[7] A. Baumbaugh, "QIE10: A new front-end custom integrated circuit for high-rate experiments," JINST, vol. 9, p. C01062, 2014.

[8] G. Dissertori et al., "Results on damage induced by high-energy protons in LYSO calorimeter crystals,"Nucl. Instrum. Meth. A, vol. 745, p. 1, 2014.
[9] To be published. Results from the Shashlik study group. The Shashlik study group consists of the following institutes: Baylor Univ., Boston Univ., Univ. California Davis, Caltech, CERN, IHEP Beijing, ETH Zurich, Fairfield Univ., Fermilab, Florida State Univ., INFN (MilanoBicocca, Roma1, Torino, Trieste), IHEP Protvino, INP (Minsk, Moscow, Tashkent), Iova, JINR Dubna, Kansas State Univ., KIPT Kharkov, ISMA Kharkov, Univ. Maryland, MEPHI Moscow, NCPHEP Minsk, Northeastern Univ., Univ. Notre Dame, Princeton Univ., Univ. Rochester, Saha Inst. Nuclear Physics, SINP Kolkata Institute, Texas Tech Univ., TIFR Mumbai Institute, Univ. Virginia.

[10] F. Pandolfi et al., Beam Test Results for a Tungsten-Cerium Fluoride Sampling Calorimeter with Wavelength-Shifting Fiber Readout, talk O12-2, this conference. 